\title{
RESUMEN
}

En sus expresiones más variadas, el arte se ha utilizado, históricamente, como una plataforma para la proyección de identidad. En América Latina, durante muchos años, se planteó la cuestión de cómo abordar las tradiciones europeas y populares sin caer en la trampa de estilizaciones vacías, carentes de contenido, y que no logran ir más allá de lo superficial. Este artículo ofrece una revisión crítica del concepto de identidad en la música latinoamericana de tradición escrita, a la luz de diferentes enfoques sociológicos, dando cuenta de la necesidad de reevaluar no solo los procedimientos técnicos de creación, sino que, además, la dimensión social, cultural e histórica que los sustenta.

Palabras Claves: identidad; sociología de la música; cultura latinoamericana; música latinoamericana, teoría cultural.

\begin{abstract}
In its most varied expressions, art has always been a platform for constructing identity. In Latin America, the question existed for many years of how to take on European and folk traditions without falling into the trap of empty stylization that lacks content and fails to go beyond the superficial. This article offers a critical review of the concept of identity in Latin American music of written tradition, under the umbrella of different sociological approaches, concluding the need to reevaluate not only the technical procedures of creation, but also the social, cultural and historical dimension that sustains them.
\end{abstract}

Keywords: identity; sociology of music; Latin American culture; Latin American music; cultural theory. 
El Concepto de Identidad. Una aproximación a la Música en América Latina The Concept of Identity. An approach to Music in Latin America

Pp. 36 a 59

\section{EL CONCEPTO DE IDENTIDAD \\ UNA APROXIMACIÓN A LA MÚSICA EN AMÉRICA LATINA}

\section{THE CONCEPT OF IDENTITY. AN APPROACH TO MUSIC IN LATIN AMERICA}

Dr. José Miguel Arellano

Universidad Adolfo Ibáñez

Chile*

\section{El Concepto de Identidad}

\section{El Caso de América Latina}

La identidad es un concepto que se aleja de cualquier definición clara y precisa. Innumerables son las páginas que se han escrito tratando de definir esta idea que parece cobrar vida de maneras muy diversas incluso entre miembros de una misma cultura. El asunto se vuelve aún más complejo cuando la música y las artes en general, se utiliza como mecanismo de reconstrucción o representación de identidad de un grupo particular de personas. Simon Frith, en su artículo Music and Identity, nos entrega una perspectiva muy interesante acerca del problema que surge a partir del estudio de la música como un objeto técnico-sonoro y la intención de articular ciertas conexiones con diferentes aspectos culturales, como en el caso de la proyección y representación de identidad. Sobre el campo específico de la música popular dirá lo siguiente:

El estudio académico de la música popular ha sido limitado por el supuesto de que los sonidos deben, de alguna manera, reflejar o representar a un 
pueblo y sus personas. El problema analítico, sin embargo, ha sido rastrear las conexiones, desde la obra, la partitura o la canción, hasta los grupos sociales que la producen y consumen. Lo que está en cuestión es la homología, una especie de relación estructural entre las formas material y musical ${ }^{1}$.

A lo largo de este trabajo nos referiremos a la identidad como un conjunto de elementos culturales, sociales y políticos que se forman a lo largo del tiempo como consecuencia de múltiples eventos de carácter históricos, políticos y sociales, y que determinan ciertas disposiciones y modos de comportamiento de un grupo específico de personas. Existen, por supuesto, muchos otros enfoques para este concepto y algunos de ellos pueden estar en el extremo opuesto de la definición que acá utilizaremos para estructurar este trabajo.

El sociólogo chileno Jorge Larraín, por ejemplo, en su libro Identidad y Modernidad en América Latina, analiza algunas de las diferentes expresiones de identidad que se dieron en el continente, categorizándolas según su situación y rol específico en el desarrollo histórico de la región. Por ejemplo, describe el movimiento indigenista de finales del siglo XIX y principios del XX, que ubica la identidad en un pasado oprimido y en la pérdida de un conjunto de tradiciones y formas de vida fuertemente vinculadas a los pueblos indígenas de la región. De esta manera, para los indigenistas, cualquier aproximación a la identidad partiría de una reconstrucción del pasado y por el redescubrimiento de las raíces perdidas, que fueron destruidas durante la invasión europea y la posterior colonización. Como diría Larraín, "su identidad está ubicada y formada, de una vez por todas, en el pasado" 2 .

Sin embargo, un elemento común, descrito por el sociólogo, en cada una de las diferentes manifestaciones de identidad que han ocurrido en América Latina es, precisamente, esta constante búsqueda de identidad. Sin importar si se entiende como un fenómeno pasado que deba reconstruirse, o como un concepto que debe articularse mirando hacia el futuro, una de las características fundamentales de las naciones latinoamericanas es esta búsqueda constante de símbolos culturales compartidos que podrían ayudar a impulsar el desarrollo artístico, político, económico y cultural de la región.

Durante las primeras décadas del siglo XIX, por ejemplo, las Guerras de Independencia significaron un período de constante conflicto para las sociedades latinoamericanas, en el que tuvieron que comenzar a definir, bajo parámetros a veces inciertos, los diversos caminos que las nuevas naciones seguirían, en términos de su construcción social y cultural.

Frith, Simon (2006). "Music and Identity", Questions of Cultural Identity. Stuart Hall and Paul du Gay ed. London: SAGE Publishers, p. 108.

2 Larraín, Jorge (2004). Identidad y Modernidad en América Latina. México D.F.: Editorial Océano de México, p. 57. 
Si analizamos aspectos puramente musicales, podemos observar que el siglo XIX es uno de los siglos decisivos no solo para el surgimiento de ciertos movimientos estético-políticos, como el indigenismo, el europeísmo y el criollismo, sino que, además, para el impacto que esos movimientos habrían de tener en la conformación de los diferentes grupos sociales de América Latina.

Con esto me refiero a la manera en que estas definiciones estéticas y políticas configuraron ciertos cursos de acción que tuvieron un efecto perjudicial en el desarrollo musical y cultural de la región como, por ejemplo, la marginación de músicas folklóricas y populares, (principalmente asociadas a los estratos socioeconómicos más desfavorecidos) la asimilación de la estética europea, y la eliminación de la cultura indígena de los discursos artísticos institucionalizados.

De esta manera, desde los primeros años de vida de las naciones latinoamericanas, después de su independencia, la construcción de una identidad se convirtió en un objeto deseado. Asimismo, la reconfiguración social del siglo XIX generó la necesidad de repensar las características culturales a través de la articulación de los más variados discursos, que pretendían dar a conocer las peculiaridades de la diversidad étnica y racial que daba forma a los diferentes países de la región. Por eso, a través de la literatura de fines del siglo XIX, por ejemplo, se exaltó el coraje de los pueblos originarios y la agilidad rítmica de las danzas africanas. Sin embargo, su valor casi exótico, residía precisamente en ser identificados como la "otredad" de lo europeo.

El musicólogo y filósofo chileno Gabriel Castillo describe en su libro Las Estéticas Nocturnas como, hacia fines del siglo XIX y comienzos del XX, a través de una gran variedad de ensayos literarios, se crea una imagen ficticia de la identidad chilena. Es posible apreciar, en consecuencia, esta necesidad de construir un imaginario colectivo no solo con el propósito de dar forma a su identidad, sino también con la idea de posicionar al país como un reflejo de la sociedad europea:

Decíamos en un comienzo que uno de los rasgos fundamentales de la representación nacional en esta segunda mitad de siglo XIX es la asimilación criolla de la identidad mapuche. Por cierto, el uso del mito araucano no cambiará un ápice, durante la república, el desprecio real de los chilenos por el Indio, cuyas virtudes eran mucho más celebradas mientras no se llevara un apellido mapuche o se pudiera ser físicamente asimilado a ellos. La apropiación histórica del Indio será mucho más fuerte mientras más permanezca éste siendo "otro"3.

Castillo Fadic, Gabriel (2003). Las Estéticas Nocturnas: Ensayos Republicano y Representación Cultural en Chile e Iberoamérica. Santiago: Frasis Editores, p. 23. 
Desde lugares aparentemente distantes, como la política y las artes, esta idea de encontrar una voz individual, de articular de diferentes maneras las formas de vida e historias de un grupo determinado de personas, se convirtió en un tema central dentro de la discusión pública. Independientemente de si esta noción de identidad se entendía, a través del discurso (artístico, político, etc.), como construcción, o como reflejo de algo previamente constituido, lo cierto es que su presencia, hacia fines del siglo XIX, se sintió cada vez con mayor intensidad.

\section{La Construcción de una Nación: ¿Cómo se Relacionan Música y Política?}

Numerosos músicos, artistas y teóricos de las ciencias sociales se han referido a la capacidad de la música y las artes en general, para articular ideas que puedan ir más allá de una dimensión puramente técnica. Es por esto que la política ha sido parte integral de diversos discursos estético-musicales que, con mayor o menor éxito, han intentado dar vida a conceptos que, en apariencia, parecieran ser ajenos a la música y a la actividad artística en general.

Recordemos, por ejemplo, las observaciones que hace el compositor alemán Helmut Lachemann sobre la necesidad de reevaluar, estéticamente, las consecuencias de la Segunda Escuela de Viena, analizando, principalmente, el concepto de belleza y como este ha sido utilizado para desviar al arte de su faceta política y transformadora, con un rol activo en la reconfiguración de la sociedad actual:

Se necesitó urgentemente tal evaluación, para aclarar la distinción entre la demanda legítima y profundamente arraigada de la humanidad por el arte como una experiencia de la belleza, y su falsa satisfacción y alienación en la forma de un falso 'forraje', fabricado por la burguesía y preservado en una sociedad de contradicciones reprimidas ${ }^{4}$.

En este sentido, la relación que existe entre la música y una dimensión política se puede analizar desde dos puntos de vista que, aunque estrechamente relacionados entre sí, hablan sobre diferentes aspectos de la construcción cultural de una sociedad. En el caso de América Latina, a pesar de las particularidades de cada nación, estas dos formas de abordar la relación que existe entre música y política ofrecen un enfoque analítico que puede ayudarnos a arrojar luz sobre el estudio de la construcción de una identidad musical dentro de la región. Me referiré a estas dos perspectivas como; a) Configuración Institucional y b) Carácter Político de una Obra Musical.

4 Lachenmann, Helmut (1980). "The Beautiful in Music Today", Tempo New Ser, 135, p. 20. 


\section{a) Configuración Institucional}

Si revisamos la historia musical de América Latina hacia fines del siglo XIX y comienzos del XX, podemos ver que en diferentes países se realizó un proceso similar para establecer los fundamentos culturales y musicales de la región, replicando lo que se había construido en Europa. En consecuencia, la mayoría de las naciones comenzaron a crear escuelas de música, sociedades musicales, orquestas sinfónicas y salas de conciertos. Toda esta arquitectura cultural e institucional tuvo el propósito de contribuir a la formación de las nuevas naciones, de acuerdo con las ideas de modernidad representadas por el viejo continente.

En el caso de Chile, por ejemplo, es posible observar la creación del Conservatorio Nacional en 1850, la Sociedad Bach en 1924 y la Orquesta Sinfónica Nacional en $1940^{5}$. De esta manera, con el propósito de replicar a Europa en América Latina, como mencionamos anteriormente, este aparato institucional operó desde la marginación y eliminación de ciertas músicas populares y folclóricas.

Para el escritor y pensador cubano Alejo Carpentier ${ }^{6}$, este proceso de institucionalización, que se estructuró a partir de la lógica cultural europea, supuso un rechazo de los valores nacionales, vale decir, para construir culturalmente las naciones de América Latina, aquellas músicas que eran ajenas a Europa fueron marginadas del discurso oficial y académico.

Para hacer frente a esta marginalización de músicas no-occidentales, los movimientos nacionalistas, hacia fines del siglo XIX y comienzos del XX, intentaron integrar lo folclórico y lo popular con elementos de la música europea, con la finalidad de hacerlos parte de este aparato institucional. Sin embargo, esta traducción y reformulación de ciertos aspectos específicos de la música popular y folclórica se llevó a cabo, en una gran mayoría de los casos, bajo la óptica estética de occidente, creando obras que, debido a su naturaleza híbrida, es difícil entenderlas como representación de una identidad.

En otras palabras, estas músicas serían una construcción de identidad sin una posición dentro del espacio social. Nos referiremos en detalle a esta idea en la última sección de este artículo, que discute la relación entre música y sociología.

Memoria Chilena (2018). "Conservatorio de Música", visitado el 01 de febrero del 2018. http://www. memoriachilena.cl/602/w3-article-93313.html.

6 Carpentier, Alejo (2004). "La Hora Actual de la Música en América Latina", América Latina en su Música. Isabel Aretz (editor). Buenos Aires, Argentina: Siglo XXI Editores S.A., pp. 7-19. 


\section{b) Carácter Político de una Obra Musical}

La música (y las artes en general) entrará en una relación dialéctica con una dimensión política cada vez que manifieste, a través de diferentes procedimientos, ya sean técnicos, narrativos o estructurales. Las contradicciones de una configuración cultural o institucional, es decir, cuando se convierta en un vehículo al servicio de develar los cimientos que estructuran la lógica estética, social y cultural de una sociedad determinada.

Thierry de Duve, teórico del arte de origen belga, identifica, no obstante, un tránsito histórico (que es posible de ser observado en el escenario cultural y artístico institucionalizado de la América Latina decimonónica) mediante el cual el arte va siendo despojado de su dimensión relacional, a nivel cultural, político y social, para erigirse como un mero objeto de apreciación estética. Por esto, en su libro Aesthetics at Large: Art, Ethics, Politics señala lo siguiente;

De la manera más honesta y directa que puedo, he aquí una visión que, espero, estimule una reflexión sobre una idea occidental moderna acerca del arte, que es, por supuesto, aquella sobre su autonomía. Aunque, en algún sentido, esta idea es infinitamente más antigua que la era moderna, $\mathrm{y}$, probablemente, no restringida solamente al mundo occidental, fue llevada a su punto culmine con la creación y desarrollo del museo, donde ciertas cosas, ya llamadas arte, fueron desligadas de su función mágica, religiosa o política, para ser almacenadas en espacios públicos consagrados al goce estético y a la contemplación desinteresada ${ }^{7}$.

Esta idea de una dimensión política del arte es desarrollada in extenso por el filósofo alemán Walter Benjamin en su obra El Autor como Productor ${ }^{8}$.

En este trabajo, Benjamin intenta redefinir la figura y rol del artista dentro de la sociedad, atribuyéndoles un papel central en la configuración de una nueva forma de entender el arte, el proceso de producción y la lucha de clases. Asimismo, se aborda la necesidad de una comprensión de la actividad artística no solo desde su base ideológica, sino también como un mecanismo de transformación social; como un vehículo de revolución. De lo contrario, dirá Benjamin, cualquier intento de transformación puede dar como resultado una contrarrevolución.

Para explicar sus ideas sobre el papel del arte y la posición del artista dentro de la sociedad, el filósofo acuña el concepto de refuncionalización, término que puede ser entendido desde diferentes perspectivas. Por un lado, implica

De Duve, Thierry (2019). Aesthetics at Large: Art, Ethics, Politics, Chicago: Chicago University Press, p. 54.

8 Benjamin, Walter (1998). Understanding Brecht. Traducido por Anna Bostock. Londres: Verso. 
repensar la situación social de la obra de arte en sí misma y su propósito dentro del aparato de producción en masa, reflejo de la sociedad capitalista dominante, $\mathrm{y}$, por lo tanto, otro mecanismo de opresión.

Por otro lado, también tiene como objetivo redefinir la función social del artista como individuo que desempeña un rol importante en la configuración social. Obviamente, autor y obra son dos objetos que están directamente relacionados, sin embargo, parece importante notar la distinción que establece Benjamin en su refuncionalización de la actividad artística.

En primer lugar, dirá el filósofo, tenemos al artista que entretiene y que se convierte en un eslabón más dentro de la cadena de producción en masa que domina la escena cultural en nuestros días. En segundo lugar, está el artista que, como Bertolt Brecht, se opone al estado de estancamiento de los procedimientos creativos, adormecidos y contaminados por el sistema capitalista, y propone una ruptura con esta forma de concebir la obra de arte, dando al "espectador" una participación en el proceso artístico y creativo.

Así es como se erige la relación entre calidad y tendencia política, como una de las piedras angulares del cambio de paradigma que Benjamin propone. Y la pregunta clave, es el papel que tiene la obra de arte dentro del proceso de producción y su contribución a la reconfiguración de una nueva forma de pensar sobre ella. Para él, esta tensión producida entre la tendencia política y la calidad se resuelve mediante el desarrollo de la técnica, es decir, a través del progreso de los elementos que forman la base del lenguaje artístico (literario, musical, etc.).

Como las condiciones sociales están profundamente determinadas por los procesos de producción actuales, para Benjamin, es indispensable una ruptura con el statu quo. El artista debe jugar un papel clave en la construcción de nuevos procedimientos técnicos y de producción, lo que, según él, tendrá un impacto positivo dentro de la reconfiguración social que busca desvincularse del capitalismo cultural que domina la sociedad actual.

Estas ideas de Benjamin, sobre el rol del artista y su obra dentro de la sociedad, nos ayudan a analizar en mayor profundidad la situación cultural y musical de América Latina. Si entendemos su configuración institucional y sus consecuencias políticas y estéticas, como una estructura de poder que no permite que aflore la esencia cultural de la región, es necesario, a través de las artes, replantearse sus mecanismos operacionales.

Los movimientos nacionalistas de finales del siglo XIX y principios del siglo XX pasaron por alto esta idea de tratar de reconstruir una posible identidad local haciendo visible aquello que fue invisibilizado. Los principios reguladores de la estética musical de occidente impidieron una verdadera comprensión de 
las realidades culturales de la región: lo inestable se estabilizó, lo irregular fue regularizado, y lo no-temperado se temperó.

\section{La Construcción de una Identidad}

Hablar sobre la identidad de una nación implica algunos problemas que no son fáciles de evitar. En primer lugar y dadas las condiciones de las sociedades modernas, es necesario tener en cuenta las diferencias en las formas de vida de los miembros de cualquier grupo analizado. Si hablamos de un país, y analizamos las estructuras culturales de, por ejemplo, lo que se conoce como clase alta y todas las implicaciones que esto conlleva, y las comparamos con las de una clase más modesta, es probable que encontremos más diferencias que similitudes.

Por lo tanto, es esencial abordar el análisis partiendo por los procesos históricos y sociales compartidos por los diferentes miembros de una comunidad, ya que sólo de esta manera será posible establecer diferentes mecanismos de reafirmación o reconstrucción de la identidad, atendiendo, en mayor o menor medida, a los diversos elementos que la constituyen.

En el caso específico de América Latina, podemos ver cómo se ha ido construyendo en el imaginario colectivo la idea que las sociedades que la componen pueden ser encapsuladas bajo los mismos parámetros descriptivos, otorgándoles un sello de identidad que, en consecuencia, se limita a describir sólo sus componentes más superficiales.

Indudablemente la historia compartida de los diferentes países de la región ha generado una multiplicidad de elementos comunes que en ningún caso pueden quedar fuera del análisis. Diferentes ejemplos de este proceso histórico compartido se pueden rastrear a lo largo de la historia: la conquista y colonización de América, los movimientos de independencia de principios del siglo XIX, los procesos de modernización y configuración institucional de principios del siglo $\mathrm{XX}$, y los períodos dictatoriales de mediados del siglo pasado'.

Sin embargo, para el escritor argentino Walter Mignolo ${ }^{10}$ lo que define con mayor claridad la construcción cultural de las naciones latinoamericanas es lo que él llama la herida colonial; una serie de consecuencias generadas a raíz de un largo período de dominación, que pueden ejemplificarse, por nombrar

9 Larraín, Jorge (2004). Identidad y Modernidad en América Latina. México D.F.: Editorial Océano de México, p. 57.

10 Mignolo, Walter (2007). La Idea de América Latina: La Herida Colonial y la Opción Decolonial. Traducido por Silvia Jawerbaum y Julieta Barba. Barcelona: Editorial Gedisa S.A., p. 34. 
sólo algunas, en temas como el racismo, el maltrato y descuido de los grupos indígenas y una búsqueda obsesiva de referentes culturales extranjeros. Todo esto, sumado a una dominación política, económica y cultural, ha derivado en el complejo escenario de identificación, compartido por los diferentes miembros de la región. Como resultado de esta herida colonial a la que alude Mignolo, se ha producido una división entre las distintas capas de la sociedad latinoamericana, separando cada una de las culturas que la habitan y haciendo del proceso de reintegración una tarea muy difícil.

\section{Música "Clásica" en América Latina}

Las artes en general, pero particularmente la música, siempre han sido utilizadas como un catalizador para los diferentes procesos sociales y culturales. A mediados del siglo XX, por ejemplo, y con el advenimiento de los diversos regímenes militares de América Latina, surgió uno de los movimientos musicales más característicos de la región: el Nuevo Canto Latinoamericano que, a pesar de sus múltiples variantes en los diferentes países, mantuvo ciertos principios compartidos por todos, como el uso de los ritmos folclóricos, el rescate de instrumentos autóctonos, una marcada ideología de izquierda y una visión crítica que apuntaba a un cambio revolucionario tanto en lo social como en lo cultural.

Muchos ejemplos como el anterior, pueden darse con respecto a la música popular y su papel en la conformación de la identidad dentro de las diferentes naciones de América Latina: el rock en Argentina, la cumbia en Colombia y gran parte de América del Sur, y el bolero en Cuba y México son sólo algunos ejemplos, de cómo la mixtura entre diferentes culturas ha sido posible. Ahora bien, ¿Cómo se puede generar una estética musical que, a la luz de los elementos constitutivos de una cultura particular, pueda concebirse a través de los mecanismos técnicos y estéticos de otra? Como siempre, es necesario entender los diferentes procesos históricos que han dado forma a las diferentes naciones de la región.

El patrimonio cultural de lo que hoy conocemos como América Latina es una consecuencia de la mezcla de tres grupos raciales, a saber, indígenas, africanos y europeos, algo que se denomina, frecuentemente, como sincretismo. Es evidente, sin embargo, que en una situación de dominación las características propias del grupo subyugado estarán siempre en una posición de desmedro con respecto a la del dominador.

En términos puramente cuantitativos, esta situación de subyugación condujo a una neutralización casi absoluta de los elementos musicales típicos de las culturas indígenas y africanas, ya que se oponían totalmente a los principios básicos del pensamiento artístico europeo, que en música se expresaron a través de simetría, consonancia y homogeneidad rítmica entre muchos otros. 
Uno de los mayores problemas que ha enfrentado la música clásica, en esta búsqueda de integración cultural, es que normalmente da forma a sus preocupaciones estéticas desde un punto de vista teórico y no desde lo que podríamos denominar como procesos artísticos orgánicos. Así, en la mayoría de los ejemplos en los que es posible observar un cruce cultural entre, por ejemplo, una cultura indígena y música europea, la aproximación se hace desde una perspectiva académica, sin haber experimentado previamente la música indígena, entendiendo la naturaleza de cada uno de los elementos que la constituyen.

Esta forma de abordar la mixtura entre lo europeo y lo vernáculo, en el caso de la música de tradición escrita, difiere con gran parte de los ritmos populares que nacieron en la región, como la cumbia, el bolero, el huayno, la zamba, la milonga y la cueca, que han evolucionado a través de los años y, en su gran mayoría, desde la periferia del aparato institucional que describimos anteriormente: en un comienzo a través de grupos indígenas y africanos, y posteriormente, en manos de campesinos, trabajadores y jóvenes marginados. Por lo tanto, para crear un diálogo entre identidad nacional y musical, se necesita mucho más que la conjunción de elementos técnicos provenientes de diversas culturas. Es necesario abordar esto no solo desde una perspectiva teórica, sino también desde la experiencia, tomando en consideración el contexto en que se desenvuelven y se han desarrollado cada una de estas culturas, así como también tomar en cuenta los procesos históricos que las han configurado, para comprender en mayor profundidad cada uno de sus elementos constitutivos.

\section{Música Post Independencia}

Las revueltas independentistas de las naciones latinoamericanas, que comenzaran con la emancipación haitiana entre los años 1790 y 1804, generaron un gran conflicto para la organización política y cultural de la región, lo que se vio reflejado, de diferentes maneras, en la producción local de música y otras formas de arte. A diferencia de lo que ocurrió durante la colonia, período en el cual hubo un gran florecimiento de un repertorio local, y del cual hemos heredado una gran variedad de fuentes primarias que pueden dar cuenta de la situación musical en ese momento, "el siglo XIX se nos presenta como una especie de edad media, de la cual ha sobrevivido muy poca música escrita por compositores locales"11.

Como en cualquier situación histórica, las razones de esta escasez de fuentes musicales primarias son múltiples y de naturaleza variada. Por ejemplo, la

11 Miranda, Ricardo y Tello, Aurelio (2001). Historia de la Música en Latinoamérica. Ciudad de México: Dirección General del Archivo Diplomático, p. 27. 
independencia de Europa condujo, durante los años siguientes a problemas políticos y económicos, y a diversos conflictos bélicos que desestabilizaron enormemente la región, y que no permitieron el desarrollo continuo de una cultura artística local. Si tomamos como ejemplo el caso de Chile, que luchó por su independencia entre los años 1810 y 1818, veremos a lo largo de su historia decimonónica, un gran número de conflictos internos y externos, a saber, guerra contra el Virreinato del Perú (1813), contra la Confederación Perú-Bolivia (18361839), contra España (1864-1866), la Guerra del Pacífico contra Perú y Bolivia nuevamente (1879-1884) y, hacia finales de siglo, la Guerra Civil de $1890^{12}$.

A pesar de ser un período en el cual se creó y conservó poca música local, esta necesidad de definición identitaria, sumada al creciente espíritu nacionalista y la caída gradual de las estructuras coloniales, se convirtió en un incentivo para desarrollar y organizar, de manera sustantiva, una gran actividad artística, a través de la creación de conservatorios, teatro y salas de ópera, que se establecieron como centros para músicos nacionales e internacionales. Ejemplos de este gran impulso cultural se pueden encontrar en todo el territorio latinoamericano, como en el caso del Conservatorio Nacional y el Teatro Municipal de Santiago de Chile, inaugurados en 1850 y 1857 respectivamente, el Gran Teatro de Córdoba, Argentina, en 1873, la Academia Nacional de Música de Colombia en 1882, y el Teatro Amazonas, de Brasil, en 1884 entre otros.

Sin embargo, no será hasta los años 30 y 40 del siglo XX que los compositores comenzarán a reevaluar las nociones básicas de su arte. Como resultado de un entorno social en constante tensión, claramente representado por la Revolución Mexicana, comienza un período de reflexión sobre la historia de América Latina. En consecuencia, aunque de manera incipiente, la música comienza a experimentar ciertos cambios estéticos en manos de compositores en diferentes países de la región, un cambio que irá en aumento conforme nos acercamos al siglo XXI.

\section{Hacia una Sociología de la Música en América Latina}

Si asumimos que la música tiene una capacidad para transmitir ideas que superen aspectos puramente técnicos, es necesario dar cuenta de los elementos sociales, políticos y culturales que la sustentan. Es, por lo tanto, de suma importancia elaborar mecanismos con los cuales aproximarse a estos elementos que se esconden debajo de la superficie, a fin de comprender, más exhaustivamente, los códigos específicos de un grupo particular de personas.

Dentro de la sociología de la música existen diversas visiones sobre cómo

12 Góngora, Mario (1981). Ensayo Histórico Sobre la Noción de Estado en Chile en los Siglos XIX y XX. Santiago: Ediciones La Ciudad, p. 9. 
abordar la tensión que existe entre su dimensión técnico-formal y los objetos sociales y culturales a los cuales se propone representar. Para Ivo Supicic, por ejemplo, el estudio de la historia de la música difiere de una sociología de la música en cuanto a que esta última considera los elementos musicales no sólo como una representación de lo social, sino como un elemento social en sí mismo:

La sociología de la música se preocupa no sólo de la típica relación entre hechos artísticos musicales y hechos sociales extra-musicales, sino también de estos mismos hechos musicales como hechos sociales. Lo musical y lo social no deben ser opuestos, ya que se interpenetran mutuamente. Muchos hechos musicales implican, en efecto, aspectos sociales ${ }^{13}$.

Desde nuestro punto de vista, el enfoque de Supicic puede llevarnos a malinterpretaciones de la realidad social que subyace a una representación musical. Si damos por evidentes los elementos técnicos, podríamos perder de vista las contradicciones que emergen entre el objeto social y el artístico, uno de los problemas que no logró sortear con éxito gran parte la estética nacionalista, al menos durante la primera mitad del siglo XX.

Un caso ejemplar de esto es el estudio realizado por Morgan James Luker, en su libro The Tango Machine ${ }^{14}$, en el cual describe el tango argentino contemporáneo a partir de las contradicciones surgidas durante la segunda mitad del siglo, en donde, por un lado, todavía se le veía como un referente cultural, especialmente en el imaginario colectivo, pero, por otro, ha perdido toda presencia real en la vida cotidiana de los argentinos, percibiéndose más como una marca nacional que como un objeto de carácter artístico. Es por esto que una sociología de la música debe guiar los intentos de reflejar una identidad con el propósito de evitar falsas representaciones.

Motti Regev, sociólogo de origen argentino, observa en el campo de la sociología de la música un terreno fértil y poco explorado, que puede arrojar luz sobre la función que cumplen los sonidos, canciones, obras, diferentes géneros, etc., en las múltiples áreas de la cultura e interacción social.

En su artículo; Musical Cosmopolitanism, Bodies and Aesthetic Cultures ${ }^{15}$ analiza éste y otros aspectos, identificando en la disciplina sociológica una herramienta extremadamente útil para la investigación socio-musical:

En primer lugar, quiero abordar la relación entre la sociología de la música y el conocimiento sociológico en general. Estas relaciones se caracterizan

3 Supicic, Ivo (1987). A Guide to the Sociology of Music. Stuyvesant, NY: Pendragon Press, p. 46.

14 Luker, Morgan James (2016). The Tango Machine. Musical Culture in The Age of Expediency. Chicago: Univ. Press

15 Smudits, Alfred ed. (2019). Roads to Music Sociology. Munich, Germany: Springer, p. 80. 
por una forma de influencia, inspiración y encuadre. Es decir, la sociología de la música está enmarcada, inspirada e influenciada por el conocimiento sociológico general, por conceptos y teorías formulados y desarrollados en diversos campos y áreas de la sociología. Que yo sepa, la dirección opuesta de inspiración e influencia apenas existe. La aplicación e implementación del conocimiento en el contexto de la sociología con el propósito de teorizar en general y conceptualizar el conocimiento sociológico apenas existe. Una excepción notable es la noción ${ }^{16}$.

El enfoque analítico de la teoría crítica sobre la sociología ha abierto nuevas puertas para comprender el papel de la disciplina no solo en lo que se refiere a la investigación puramente social, sino que también en términos de una posible reevaluación de los propios mecanismos que los sociólogos utilizan para dar forma a sus investigaciones.

Una de las críticas más fuertes de esta escuela, ha estado dirigida hacia el cientificismo extremo con el que la sociología ha llevado a cabo sus investigaciones, sin superar lo meramente descriptivo para articular un análisis crítico de las estructuras sociales contemporáneas. Por lo tanto, con sus raíces en la teoría marxista y su enfoque principal en la dominación económica, la teoría crítica ha recurrido al análisis de varias formas de interacción social, dentro de las cuales la cultura se destaca como uno de los mecanismos más evidentes para la dominación. Esta aproximación se nos presenta como una gran oportunidad para descubrir las relaciones que se establecen entre la música, como un conjunto de elementos técnicos y artísticos, y una posible dimensión conceptual y simbólica.

En el caso de la música latinoamericana de tradición escrita, utilizada como vehículo para la representación y proyección de identidad, sería suficiente estudiar las relaciones de poder entre las diferentes culturas en juego para entender estas estructuras de dominación. La historia nos ha demostrado que los rasgos específicos que podrían definir la identidad de una cultura, generalmente se filtran a través de la óptica de estética europea. Si pensamos en la música de compositores como Manuel Ponce o Heitor Villa-Lobos, veremos que el uso de material folklórico e indígena ha sido extremadamente estilizado, con la finalidad de adaptarse a los estándares musicales de la tradición occidental. Sería injusto, sin embargo, no valorar su contribución al desarrollo de una conciencia latinoamericana que ha dirigido su mirada hacia grupos cuyas culturas y formas de vida han sido sistemáticamente marginadas $\mathrm{y}$, en algunos casos, definitivamente eliminadas.

16 Smudits, Alfred ed. (2019). Roads to Music ..., p. 80. 
¿Identidades Nacionales o Sociales?

Uno de los puntos centrales en la discusión sobre la identidad, y sus diferentes manifestaciones a través de las artes, tiene que ver con el tema de la representación. Si hablamos de identidad a través del sonido, esto debe ser un reflejo de algo previamente constituido y compartido por miembros de una comunidad determinada. De lo contrario, esta supuesta proyección de identidad se convertiría en una construcción sin un correlato dentro del tejido social. Si bien es evidente, lo anterior es fundamental para poder articular un discurso que, con el objetivo de comprender el fenómeno de la identidad musical, tenga en cuenta la realidad cultural y social, dentro de la cual se desarrolla una determinada obra, así como sus implicaciones en el ámbito de lo representacional, es decir, en cómo los diferentes contextos determinan, en mayor o menor medida, un conjunto de elementos de carácter técnico y sonoro.

Por lo tanto, es importante no perder de vista el hecho de que cada uno de los diferentes estratos sociales que componen una sociedad responden a códigos culturales y simbólicos específicos, que desde un punto de vista histórico son parte integral de su desarrollo como grupo social.

En el Chile del siglo XIX, por ejemplo, tenemos al mundo campesino asociado con el desarrollo de la tonada y otras formas musicales folclóricas, así como a las élites económicas y políticas con el cultivo de la música europea y todas las instituciones creadas para replicar el modelo cultural del viejo continente.

Si atendemos, musicalmente hablando, a la especificidad de cada uno de los grupos que conforman una sociedad determinada, el concepto de identidad se vuelve aún más complejo. A través de la música, es posible establecer conexiones con varios elementos de naturaleza simbólica que nos permiten elaborar ciertas nociones de identidad. De la misma manera, la adscripción cultural y social a una práctica musical sobre otra, define ciertas características individuales y colectivas. Dice el musicólogo inglés Nicholas Cook:

En el mundo de hoy, decidir qué música escuchar es una parte importante de decidir y anunciar a las personas no solo quién quieres ser, sino quién eres [...] La música es una palabra muy pequeña para abarcar algo que denota tantas formas como identidades culturales o subculturales existentes ${ }^{17}$.

En América Latina, los intentos de construir una identidad local, en las décadas posteriores a las guerras de independencia, hicieron visible una multiplicidad de problemas de naturaleza cultural, algunos de ellos sin resolver

17 Cook, Nicholas (1998). Music: A very Short Introduction. Oxford University Press, p. 5. 
hasta el día de hoy. La gran diversidad racial y cultural de las nuevas naciones hizo muy difícil articular un discurso artístico que pudiera tener sentido y tomar en consideración con la misma profundidad cada una de sus capas sociales.

¿Cómo puede ser posible, entonces, dar cohesión social y cultural a grupos tan diferentes como los provenientes de África y Europa con diversos grupos aborígenes? ¿O desde un punto de vista musical, cómo lograr una convivencia orgánica entre la complejidad rítmica, melódica y sonora de la música indígena, tomando en cuenta su dimensión espiritual, con la racionalidad, el formalismo y el equilibrio de la música de Europa?

La búsqueda de identidades nacionales se ha llevado a cabo, en gran medida, mediante la apropiación y estilización de objetos culturales provenientes de los grupos sociales marginados, eliminando todo sentido de funcionalidad y suprimiendo su dimensión simbólica original.

Entre los numerosos análisis que se han hecho sobre la dimensión sociopolítica de la música, las ideas de Theodor Adorno ${ }^{18}$ son las más relevantes y familiares de todas. Para el filósofo alemán, la música no debe simplemente reflejar los problemas sociales de un período determinado, sino que ir más allá, asumiendo un papel fundamental de oposición a las estructuras capitalistas que configuran la situación actual de las sociedades, algo que él ejemplifica, principalmente, en la cultura de masas y su desvinculación con la realidad histórica y sus procesos sociales. El sociólogo francés Pierre Bourdieu ${ }^{19}$ realizó una de las investigaciones más profundas sobre esto último, intentando establecer una conexión entre el consumo cultural y la posición del consumidor dentro del espectro social.

Este estudio, en el que se entrevistó a un gran número de personas de diferentes estratos sociales, tuvo como objetivo reevaluar la teoría estética de $\operatorname{Kant}^{20}$ (que sugería una cierta independencia de los juicios estéticos) a fin de posibilitar un análisis que tomara en consideración los características y condiciones socio-culturales de los diferentes grupos de la sociedad francesa.

De esta manera, para Bourdieu, la historia de vida de una persona tendrá una influencia directa en su capacidad para apreciar estéticamente ciertas manifestaciones artísticas y musicales, teniendo en cuenta sus propias circunstancias históricas. Tomando como punto de partida las ideas sobre el capital económico desarrolladas por Karl Marx, el sociólogo francés acuñó

18 Adorno, Theodor (1967) Introduction to Sociology of Music. Traducido por E.B. Asthon. Nueva York: Seabury Press

19 Bourdieu, Pierre. (1984) Distinction: A Social Critique of the Judgement of Taste. Traducido por Richard Nice. Cambridge, Massachusetts: Harvard University Press.

20 Kant, Immanuel. (1965) Critique of Judgement. New York: Oxford University Press. 
el término de capital cultural, "la colección de elementos simbólicos como habilidades, gustos, postura, vestimenta, manierismos, pertenencias materiales, credenciales, etc. que se adquieren al formar parte de una clase social determinada", ${ }^{21}$ para indicar la compleja conjugación de elementos que juegan un papel fundamental en la construcción de un imaginario social compartido por miembros de un mismo grupo.

De esta forma, el capital cultural ejercerá una cierta predisposición en la forma de acercarse a diversas experiencias culturales, atendiendo los códigos aprendidos por ser miembro de un determinado grupo social. Así mismo, el capital cultural se expresará a través de lo que Bourdieu llama el Habitus, "la encarnación física del capital cultural, a los hábitos profundamente arraigados, las habilidades y las disposiciones que poseemos debido a nuestras experiencias de vida" 22 .

Este enfoque analítico de las estructuras culturales de los diferentes estratos sociales y sus consecuencias en las consideraciones estéticas nos ayuda a observar, estudiar y comprender cómo el discurso nacionalista que se originó en América Latina intentó disolver las fronteras sociales entre los diferentes grupos de la región, elaborando objetos artísticos de carácter híbrido, sin posición dentro del espacio social. No hubo consideraciones importantes con respecto al valor social o el trasfondo simbólico que ciertas prácticas musicales representaban para aquellos grupos de las que surgieron, especialmente con respecto a cierta música indígena, muchas de las cuales se redujeron simplemente a un cúmulo de elementos técnicos. De hecho, una de las conclusiones que el sociólogo francés derivó de su investigación, teniendo en cuenta las peculiaridades del caso específico de Francia, tiene que ver con la fuerza que las personas en posiciones de poder podían ejercer sobre los grupos más desfavorecidos.

Por lo tanto, la construcción cultural de América Latina y el trasplante del canon artístico europeo, desde esta perspectiva institucional, no responde a ideas puramente estéticas, sino también a consideraciones políticas y sociales. Europa representó el camino a seguir para cualquier nación que deseara acercarse al desarrollo. De la misma manera, como dice Miguel Rojas Mix:

No es solo la admiración por el europeo, también es el desprecio por lo indígena. El criollo fundó su prestigio social al descender directamente del

21 New Connections to Classical and Contemporary Perscpective, Social Theory Re-Wired: Routledge, "Pierre Bourdieu: Cultural Capital", visitado el 29 febrero del 2018. http://routledgesoc.com/category/profile-tags/cultural-capital

22 New Connections to Classical and Contemporary Perscpective, Social Theory Re-Wired: Routledge, "Pierre Bourdieu: Cultural Capital", visitado el 29 febrero del 2018.

http://routledgesoc.com/category/profile-tags/cultural-capital 
conquistador, y su mayor título de orgullo era ser genética y culturalmente un blanco ${ }^{23}$.

Para el sociólogo chileno Jorge Larraín, esta disyunción social se articuló en torno a dos conceptos que resultaron antagónicos: identidad y modernidad. El primero de ellos puso sus intereses en aquellos valores y formas de vida que habían visto truncado su desarrollo como resultado de la invasión europea y sus consecuencias posteriores. La modernidad, por otro lado, era una expresión del futuro: la posibilidad de dar forma a un proyecto social, político y artístico tomando como referencia la Ilustración.

Según Larraín, esta confrontación incomprendida supuso en América Latina una lectura errónea de las implicancias del concepto de modernidad. Se entendió como un objeto exógeno que nada tenía que ver con las características y singularidades de la historia latinoamericana. Por lo tanto, como resultado de esta oposición conceptual entre identidad y modernidad, ambas fueron utilizados para organizar socialmente la región en diferentes situaciones y contextos históricos. En palabras de Larraín:

Este desplazamiento de la modernidad con respecto a la identidad no puede sino alentar la idea de que la modernidad es un injerto retrasado y mal recibido de una identidad ya constituida, o que la identidad es un obstáculo obsoleto y tradicional a una modernización indispensable [...] Las teorías de modernización son más ampliamente aceptadas en tiempos de desarrollo acelerado y expansión económica. En cambio, las teorías de identidad han surgido con mayor fuerza en períodos de crisis o estancamiento ${ }^{24}$.

\section{Capital Cultural y la Construcción de Identidad}

Para describir gráficamente el comportamiento político, artístico y cultural de la sociedad, Bourdieu estudió el espacio social, analizando la interacción entre capital económico y cultural, observando cómo se influyen mutuamente en la conformación de las estructuras que sostienen a la sociedad en sus diferentes capas. Como es posible apreciar en la Figura 1, correspondiente al espacio social descrito por el sociólogo, los comportamientos políticos, así como las preferencias artísticas, musicales e incluso deportivas, están estrechamente relacionadas con el poder adquisitivo de cada uno de los miembros de la sociedad.

23 Rojas Mix, Miguel (1991) Los Cien Nombres de América: Eso Que Descubrió Colón. San José, Costa Rica: Editorial Universidad de Costa Rica, p. 158.

24 Larraín, Jorge (2004). Identidad y Modernidad en América Latina. México D.F.: Editorial Océano de México, p. 57. 


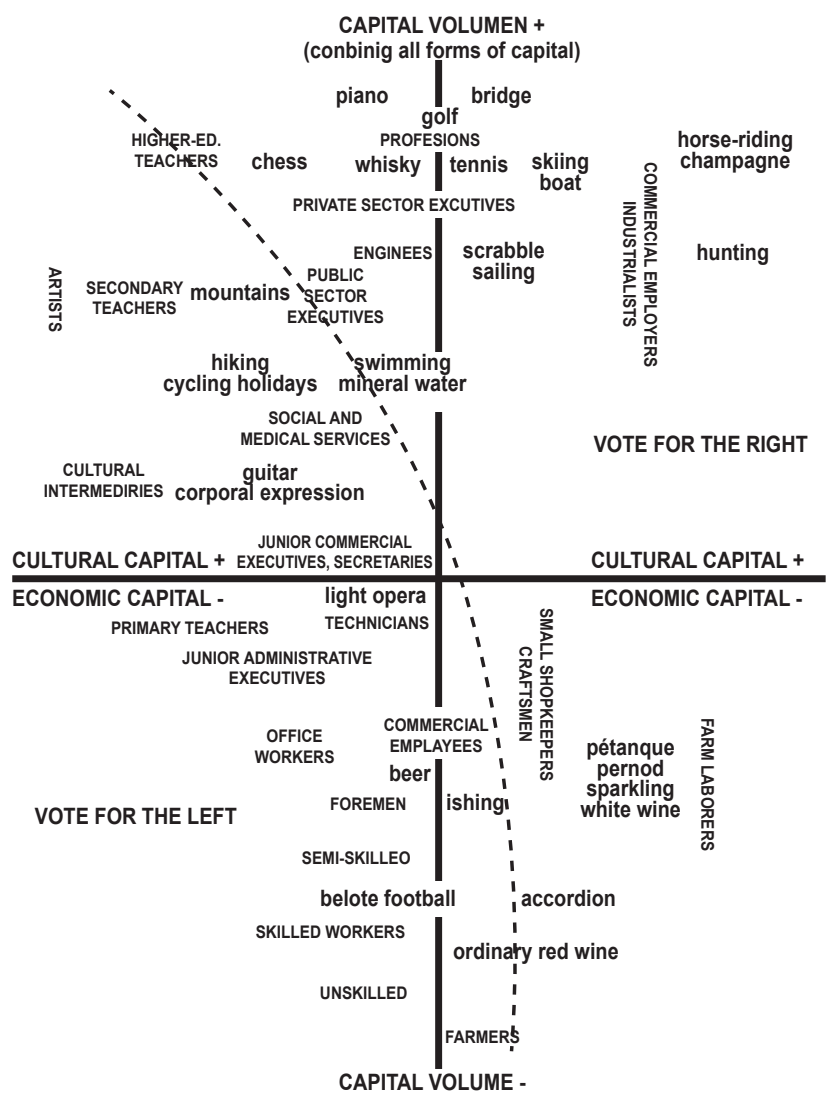

Figura 1 Espacio Social y Capital Cultural de Pierre Bourdieu ${ }^{25}$

La sección superior izquierda del gráfico, por ejemplo, correspondiente a la relación proporcional entre capital cultural + y capital cultural -, está compuesta por profesores secundarios y universitarios, músicos, escritores e intelectuales, entre otros, y dentro de sus preferencias artísticas es posible apreciar una cierta inclinación hacia la música clásica, poesía, literatura más compleja, etc. Asimismo, los miembros de este grupo corresponden, en general, a aquellas personas que han profundizado su formación académica con diversos estudios de postgrado.

Debajo de este grupo podemos encontrar a los miembros del segmento inferior izquierdo, que tienen, comparativamente, un volumen de capital menor

25 Bourdieu, Pierre (1997). Razones Prácticas: Sobre la Teoría de la Acción. Traducido por Thomas Kauf. Barcelona: Editorial Anagrama. 
a los de la esquina superior izquierda. Culturalmente, según las observaciones de Bourdieu, este segmento social, compuesto por profesores primarios, funcionarios públicos y técnicos, se expresa, culturalmente, a través de su predilección por la música popular y folclórica, la cerveza, el fútbol, etc., y, como el grupo anterior, tendría una mayor afinidad política con las ideas políticas de izquierda.

Ahora bien, si analizamos los aspectos puramente musicales, como los que se han mencionado a lo largo de este trabajo, es claro que ciertas manifestaciones se asocian con mayor claridad a un grupo social que a otros. El folclore, por ejemplo, ha sido una expresión cultural y musical, vinculada principalmente a campesinos y, en general, a la clase trabajadora, representada en el esquema Bourdieu por la sección inferior del gráfico.

Asimismo, como en el caso de América Latina, la música clásica se cultivó sistemáticamente, tanto desde la creación como desde su consumo, dentro de los grupos de clase alta, como una forma de perpetuar y reproducir ciertos códigos y símbolos culturales heredados del mundo europeo.

¿De qué manera entonces, con el mapa conceptual de Bourdieu en mente, podemos entender esta idea de identidad que surgió con tanta fuerza en el siglo XIX? Si hablamos de identidad, en una dimensión musical, como una forma de expresar sonoramente ciertas características particulares de la interacción social y las formas de vida colectivas, ¿Qué tienen en común los miembros de las clases sociales acomodadas con aquellos de clase más modesta? Tal vez sea ilustrativo recordar la observación que Miguel Rojas Mix tiene sobre este problema:

No hay costumbres nacionales, sino de clase (...) un rico español es más parecido a un inglés rico que a un español pobre. Si hay identidades de carácter popular, que se vislumbran en la vida cotidiana, en ciertos valores, en un lenguaje, en los gestos de carácter, en la música, en el folclore (...) también hay otros de las oligarquías y las burguesías emergentes (...) se puede estudiar la identidad en el discurso intelectual, o investigar en el día a día (...) también se puede decir que hay identidades atribuidas y reclamadas, ambas asociadas con la imagen (...) Las clases dominantes a menudo asumen las identidades impuestas. Encontraron su sistema de prestigio, precisamente, en el desarrollo de un 'identidad exótica', en su dépaysement, en ser culturalmente extraño en su propio mundo ${ }^{26}$.

26 Rojas Mix, Miguel (1991) Los Cien Nombres de América: Eso Que Descubrió Colón. San José, Costa Rica: Editorial Universidad de Costa Rica, p. 158. 


\section{Consideraciones Finales}

Desde la independencia de las naciones latinoamericanas, el problema con respecto a la identidad ha girado en torno a diferentes áreas de la vida pública. A través de la literatura, las artes visuales, la música y otras disciplinas, se ha intentado dar forma a diversos discursos político-culturales con la intención de develar la cara oculta de la región, a raíz años de dominio en diversas áreas, desde la artística y estética hasta la económica y política. Un enfoque multidisciplinario, desde las artes, la sociología, la antropología, los estudios de género y la historia, entre otras, puede permitirnos comprender mejor no solo la condición social de América Latina, sino también proporcionarnos las herramientas para producir los cambios necesarios para lograr una mayor integración y desarrollo dentro la región.

El propósito de este artículo no ha sido, desde luego, determinar o describir la identidad de América Latina, sino revisar ciertos enfoques prácticos y teóricos que pueden ayudarnos a comprender el fenómeno de una manera más integral. Un arte que pretenda constituirse como proyección de identidad debe, necesariamente, considerar aquellos aspectos políticos e históricos que han sido parte integral de la evolución cultural de América Latina, considerando, especialmente, aquellas imposiciones de carácter estético-teórico que han operado durante tanto tiempo dentro de la región. Asimismo, es importante considerar las ideas desarrolladas por la teoría sociológica, como las de Bourdieu, que cuestionan la noción de identidad como algo que pueda ser extrapolado indiscriminadamente a miembros de un mismo país, pero de diferentes clases sociales.

En su análisis de la situación cultural y social de Francia, Pierre Bourdieu no habla directamente del concepto de identidad, sin embargo, la ecuación entre Capital Cultural y Habitus sirve como una buena analogía para entender la evolución de la música en América Latina. Los elementos de naturaleza simbólica, como la identidad, son el resultado de ciertas disposiciones sociales y económicas, y en general no emanan de nada intrínseco al ser humano. El gusto por ciertas artes es consecuencia y resultado de una acumulación de experiencias, tales como la educación y las relaciones sociales. Por esta razón, hablar de identidades nacionales será siempre una idea que estará limitado a describir aspectos que, en su mayoría, no irán más allá de una descripción superficial $^{27}$.

27 Con esto quiero decir que esta idea de costumbres nacionales tiende a eliminar las particularidades de las diferentes clases sociales, que tienen experiencias culturales muy diferentes entre sí. Por esa razón, para Pierre Bourdieu y Miguel Rojas Mix, no habría costumbres nacionales, sino costumbres de clases. 
Es muy difícil ofrecer resultados precisos sobre cómo proceder con el proyecto de creación de América Latina. Una gran cantidad de músicos, compositores e intérpretes han tratado de contribuir a generar una conciencia sobre la creación de un arte musical que considere la realidad social y cultural de la región. Sin embargo, más allá de ofrecer soluciones y formas de proceder, nos parece más importante estar al tanto de la necesidad de redescubrir el potencial de transformación del arte latinoamericano. Es posible, a través de él, revelar los mecanismos de opresión estética, política, económica, etc., para promover el resurgimiento de una cultura verdaderamente latinoamericana. Como dice Eduardo Galeano:

La independencia es todavía una tarea por hacer, como lo fue en todas las Américas. Todas nuestras naciones nacieron engañadas. La independencia olvidó a quienes arriesgaron sus vidas por ella. Y las mujeres, los jóvenes, los indios y los negros no fueron invitados a la fiesta ${ }^{28}$.

\section{BIBLIOGRAFÍA}

Adorno, Theodor (1967). Introduction to Sociology of Music. Traducido por E.B. Asthon. Nueva York: Seabury Press, falta el número de página.

Bakers, Geoffrey and Knighton, Tess (2011). Music and Urban Society in Colonial Latin America. New York: Cambridge University Press. falta el número de página

Bourdieu, Pierre (1984). Distinction: A Social Critique of the Judgement of Taste. Traducido por Richard Nice. Cambridge, Massachusetts: Harvard University Press.

Bourdieu, Pierre (1997). Razones Prácticas: Sobre la Teoría de la Acción. Traducido por Thomas Kauf. Barcelona: Editorial Anagrama.

Benjamin, Walter (1998). Understanding Brecht. Traducido por Anna Bostock. Londres: Verso.

Benzecry, Claudio. (2011). The Opera Fanatic: Ethnography of an Obsession. Chicago: University of Chicago.

28 Daily Motion. (2011). “Entrevista con Eduardo Galeano," Telesur, visitado el 01 de febrero del 2018. http:// www.dailymotion.com/video/xhdqo6 
Carpentier, Alejo (2004). "La Hora Actual de la Música en América Latina", América Latina en su Música. Isabel Aretz (editor). Buenos Aires, Argentina: Siglo XXI Editores S.A., pp. 7-19.

Castillo Fadic, Gabriel (2003). Las Estéticas Nocturnas: Ensayos Republicano y Representación Cultural en Chile e Iberoamérica. Santiago: Frasis Editores, p. 23.

Claro Valdés, Samuel y Urrutia Blondel, Jorge (1973). Historia de la Música en Chile. Santiago, Chile: Editorial Orbe.

Cook, Nicholas (1998). Music: A very Short Introduction. Oxford University Press, p. 5.

De Duve, Thierry (2019). Aesthetics at Large: Art, Ethics, Politics. Chicago: Chicago University Press, p. 54.

De Nora, T. (2000). Music in Everyday Life. Cambridge: Cambridge University Press.

Deranty, Jean-Philippe ed. (2010). Jaques Ranciere: Key Concepts. Durham, UK: Acumen Publishing Limited.

Díaz, Rafael (2008). "La excéntrica identidad mapuche de la música chilena contemporánea: del estilema de Isamitt al etnotexto de Cáceres". Revista Cátedra de Artes, 5, pp. 65-93.

Góngora, Mario (1981). Ensayo Histórico Sobre la Noción de Estado en Chile en los Siglos XIX y XX. Santiago, Chile: Ediciones La Ciudad, p. 9.

Guénon, René (1995). The Reign of Quantity and the Signs of the Times. Hillsdale, NY: Sophia Perennis.

Frith, Simon (2006). "Music and Identity." Questions of Cultural Identity, editado por Stuart Hall y Paul du Gay, London, UK: SAGE Publishers, pp. 108127.

Isamitt, Carlos (1957). “El Folkore en la Creación Artística de los Compositores Chilenos". Revista Musical Chilena, 11, 55, pp. 24-36.

Kant, Immanuel (1965). Critique of Judgement. New York: Oxford University Press. 
Lachenmann, Helmut (1980). “The 'Beautiful' in Music Today", Tempo, 135, pp. $20-24$.

Larraín, Jorge (2004). Identidad y Modernidad en América Latina. México D.F.: Editorial Océano de México, p. 57.

Livingstone, Rodney (trans.) (1999). Walter Benjamin: Selected Writings. Cambridge, Massachusetts: Harvard University Press.

Mead, G.H. (1934). Mind, Self and Society. Chicago: University of Chicago Press.

Méndez Torellas, Gabriel, ed. (2009). Disonancias: Introducción a la Sociología de la Música. Madrid: Akal.

Mignolo, Walter (2007). La Idea de América Latina: La Herida Colonial y la Opción Decolonial. Traducido por Silvia Jawerbaum y Julieta Barba. Barcelona: Editorial Gedisa S.A., p. 34.

Miranda, Ricardo y Tello, Aurelio (2001). Historia de la Música en Latinoamérica. Ciudad de México: Dirección General del Archivo Diplomático, p. 27.

Luker, Morgan James (2016). The Tango Machine. Musical Culture in The Age of Expediency. Chicago: Univ. Press.

Regev, Motti (2019). "Musical Cosmopolitanism, Bodies and Aesthetic Cultures", Roads to Music Sociology, Alfred Smudits (editor). Munich: Springer VS. pp. 79-94.

Rojas Mix, Miguel (1991). Los Cien Nombres de América: Eso Que Descubrió Colón. San José: Editorial Universidad de Costa Rica, p. 158.

New Connections to Classical and Contemporary Perspective, Social Theory Re-Wired: "Cultural Capital: Pierre Bourdieu". Consultado el 29 febrero del 2018. http://routledgesoc.com/category/profile-tags/cultural-capital 